\title{
Koopman Reduced Order Control for Three Body Problem
}

\author{
Haoze Tang \\ John Carroll Catholic High School, Alabama, USA \\ Email: hztang2015@yahoo.com
}

How to cite this paper: Tang, H.Z. (2019) Koopman Reduced Order Control for Three Body Problem. Modern Mechanical Engineering, 9, 20-29.

https://doi.org/10.4236/mme.2019.91003

Received: November 9, 2018

Accepted: January 29, 2019

Published: February 1, 2019

Copyright $\odot 2019$ by author(s) and Scientific Research Publishing Inc. This work is licensed under the Creative Commons Attribution International License (CC BY 4.0).

http://creativecommons.org/licenses/by/4.0/

\section{(c) (i) Open Access}

\begin{abstract}
In this paper, we use a Circle Restricted Three-Body Problem (CRTBP) to simulate the motion of a satellite. Then we reformulate this problem with the controller into the description using Koopman eigenfunction. Although the original dynamical system is nonlinear, the Koopman eigenfunction behaves linearly. Choosing Jacobi integral as the Koopman eigenfunction and using the zero velocity curve as the reference for control, we are allowed to combine well-developed Linear Quadratic Regulator (LQR) controller to design a nonlinear controller. Using this approach, we design the low thrust orbit transfer strategy for the satellite flying from the earth to the moon or from the earth to the sun.
\end{abstract}

\section{Keywords}

Circle Restricted Three-Body Problem, Koopman Eigenfunction, Zero Velocity Curve

\section{Introduction}

Restricted three-body problems usually have a satellite which has a relatively small mass introduced into a system of two large masses orbiting circularly around their center of mass. CRTBP has many situations that it applies for in the solar system, such as a satellite traveling from earth to moon or comet entering our Sun-Earth system.

In order to solve the optimal control problem of the orbit transfer, a wide range of traditional optimal control strategy is employed. Traditional methods for optimal control of orbit transfer are mainly divided as the direct method [1], and indirect method. Using the framework of the direct methods, the state and the control variables are directly discretized, and the optimal control problem is converted into a nonlinear programming (NLP) problem [2]. The major direct 
methods include spectral collocation [3] [4], spline collocation [5]. Direct methods are robust and can easily accommodate path constraints, but they often require many computational resources especially for complex orbit transfer. For indirect methods, they rely on the calculus of variations, and the necessary condition of optimality requires the solution of the two-point-boundary-value-problem (TPBVP) or the Pontryagin's maximum principle. However, the disadvantage of indirect methods is often extremely difficult to solve. Another popular traditional method for optimal control is the so-called Linear Quadratic Regularization (LQR) control, whose cost function is quadratic. The quadratic cost function can be thought as the control energy.

However, neither direct method nor indirect method could provide a general solution methodology for a nonlinear optimal control problem [5] [6]. For direct method, the transcribed nonlinear programming problem may be not well-posed or converged to a local optimal solution. Especially for nonlinear problems, the collocation points will be huge to meet resolution requirements, and thus it requires large computational resources beyond current computational tools. For indirect method, the existence of the optimal solution is also questionably depending case by case.

In this paper, we use a Circle Restricted Three-Body Problem (CRTBP) to simulate the motion of a satellite. Then we reformulate this problem with controller into the description using Koopman eigenfunction. Choosing Jacobi integral as the Koopman eigenfunction and using the zero velocity curve as the reference for control, we are allowed to combine well-developed linear controller to design nonlinear control strategy. This approach is then employed to perform the optimal control on circular restricted three-body problem.

\section{Basic Theory}

\subsection{Restricted Circle Three Body Problem}

In a circular restricted three-body problem, the satellite's mass is negligible with respect to the other two, and thus we neglect the force from the third mass acting on other two larger masses. When considering a rotating reference frame, the two co-orbiting bodies are considered stationary, and the ratios of mass and distance are considered instead of their actual value because their relative value is of the most importance. The governing equations of restricted circle three-body problem are:

$$
\begin{gathered}
\frac{\mathrm{d}^{2} x}{\mathrm{~d} t^{2}}-2 \frac{\mathrm{d} y}{\mathrm{~d} t}=x-(1-\mu) \frac{x-x_{1}}{r_{1}^{3}}-\mu \frac{x-x_{2}}{r_{2}^{3}} \\
\frac{\mathrm{d}^{2} y}{\mathrm{~d} t^{2}}-2 \frac{\mathrm{d} x}{\mathrm{~d} t}=x-(1-\mu) \frac{y}{r_{1}^{3}}-\mu \frac{y}{r_{2}^{3}}
\end{gathered}
$$

where

$$
r_{1}=\sqrt{\left(y_{1}+\mu\right)^{2}+y_{3}^{2}}
$$




$$
r_{2}=\sqrt{\left(y_{1}-1+\mu\right)^{2}+y_{3}^{2}}
$$

and we set $\mu=0.012155085$ that is the mass ratio between the earth and moon. We can rewrite these formula with a substitution: $y_{1}=x, y_{2}=\dot{x}, y_{3}=y, y_{4}=\dot{y}$

$$
\left(\begin{array}{l}
\dot{y}_{1} \\
\dot{y}_{2} \\
\dot{y}_{3} \\
\dot{y}_{4}
\end{array}\right)=\left(\begin{array}{c}
y_{2} \\
2 y_{4}+y_{1}-\frac{(1-\mu)\left(y_{1}+\mu\right)}{r_{1}^{3}}-\frac{\mu\left(y_{1}-1+\mu\right)}{r_{2}^{3}} \\
y_{4} \\
-2 y_{2}+y_{3}-\frac{(1-\mu) y_{3}}{r_{1}^{3}}-\mu
\end{array}\right):=f(y)
$$

Jacobi's integral is the only known conserved quantity for the circular restricted three-body problem. This integral is used to derive numerous solutions in special cases of three body problem. The Jacobi integral remains constant even though Energy and momentum are not conserved separately, and is expressed as follow in the $(x, y)$-coordinate system:

$$
C=\left(x^{2}+y^{2}\right)+2\left(\frac{1-\mu}{r_{1}}+\frac{\mu}{r_{2}}\right)-\left(\dot{x}^{2}+\dot{y}^{2}\right)
$$

The zero-velocity surface relates to the restricted three body problem of gravity and it plays a critical role in restricted circle three-body problem. It represents a surface that a body of given energy cannot cross through, as it would have zero velocity if reaching the surface. As is shown in Figure 1, Zero velocity curve that passes through $L_{1}$ Lagrangian point represents the surface that the third body flying at rest from the left of $L_{1}$ cannot cross. Thus, the third body needs to move around the largest body permanently. However, the zero velocity contour that passes through $L_{2}$ is much more interesting and we can see that the third body

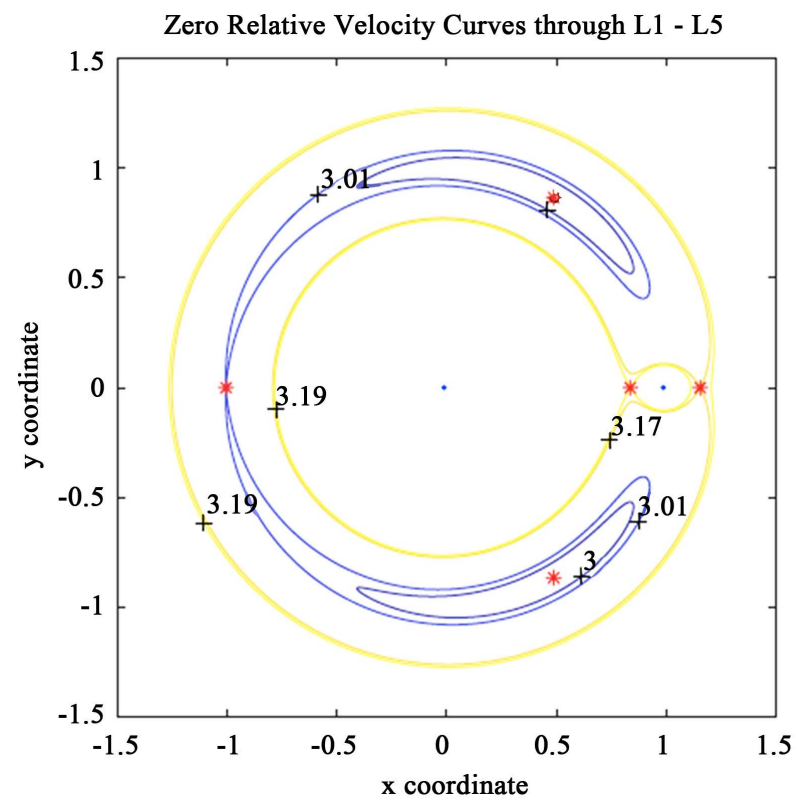

Figure 1. Zero velocity that pass through $L_{1}$ to $L_{5}$. 
can transfer between the largest body and the second largest body. This zero velocity curve provides us the potential that controlling the Jacobean (that include both kinetic energy and potential energy) will control the orbit transfer between these two large bodies. In what follows, we utilize this difference to move the satellite from orbits around the earth to the orbits around the moon, where we denote the larger two bodies as earth and moon while treating the third body as satellite.

\subsection{Koopman Eigenfunction}

We write the governing equation of the three body problem in this generalized form:

$$
\frac{\mathrm{d}}{\mathrm{d} t} y(t)=f(y)
$$

And then we are going to define its corresponding Koopman eigenfunction. In 1931, B. O. Koopman [7] introduced the operator theoretic perspective, showing that there exists an infinite dimensional linear operator $K_{t}$ that acts to advance all measurement functions $g$ of the state with the flow of the dynamics. For the infinite dimensional linear operator $K_{t}$, its eigenfunction is of the most importance and it satisfies the following equation [5] [8] for a continuous system:

$$
\frac{\mathrm{d}}{\mathrm{d} t} \phi(y)=\lambda \phi(y)
$$

The Koopman eigenfunction can be incorporated into the original governing Equation (7) using the chain rule, where it gives:

$$
\frac{\mathrm{d}}{\mathrm{d} t} \phi(y)=\nabla \phi(y) \cdot \dot{y}=\nabla \phi(y) \cdot f(y)
$$

With the definition of the Koopman eigenfunction, the eigenfunction satisfies:

$$
\nabla \phi(y) \cdot f(y)=\lambda \phi(y)
$$

Furthermore, we can implement Koopman eigenfunction into a dynamical system with control. For example, we add the thrust force in three body problem acting directly on $x$ and $y$ direction acceleration.

$$
\frac{\mathrm{d}}{\mathrm{d} t} y=f(y)+B u
$$

where $u$ is the input and $B$ is the corresponding matrix:

$$
B=\left[\begin{array}{ll}
0 & 0 \\
1 & 0 \\
0 & 0 \\
0 & 1
\end{array}\right], u=\left[\begin{array}{l}
u_{x} \\
u_{y}
\end{array}\right]
$$

This linear system represented in Koopman eigenfuction is:

$$
\frac{\mathrm{d}}{\mathrm{d} t} \phi(y)=\nabla \phi(y) \cdot \dot{y}=\nabla \phi(y) \cdot[f(y)+B u]=\lambda \phi(y)+\nabla \phi(y) \cdot B u
$$


In this form, the orignial nonlinear system $f(y)$ in the governing equation is transformed into a linear one, which is represented as the eigenvalue. The control input enters the dynamics of Koopman eigenfunction $\phi$ via an additional term which is linear in $\phi$ but possibly nonlinear in control.

Because the Jacobi integration is the conservation quantity of restricted three body problem, it corresponds to a Koopman eigenfunction with eigenvalue $\lambda=$ 0, i.e.:

$$
\frac{\mathrm{d} C}{\mathrm{~d} t}=0 \times C
$$

Thus, we use Jacobi integration of restricted three body problem as Koopman eigenfunction for the reduced order optimal control.

Although there are plenty of possible Koopman eigenfunctions, Jacobean integral is the known one that satisfies the definition of Koopman eigenfunction exactly. Setting velocity in Jacobean integral as zero, it also provides the physical meaning; i.e., zero velocity curve that serves to represent the potential energy of the system. In the following work, we are also going to utilize the zero velocity curve as the guidance for the control reference of Koopman eigenfunction.

\subsection{Combining with Linear-Quadratic Regulator}

With Koopman eigenfunction, we have transformed the dynamics in the system of $\phi$, which is linear. This offers us the space to utilize the traditional linear quadratic controller [5].

$$
J(\phi, u)=\frac{1}{2} \int_{0}^{1} \phi^{\mathrm{T}} Q \phi+u^{\mathrm{T}} R u
$$

where $\phi$ is the Koopman eigenfunction for our problem; i.e. Jacobean integral in restricted three body problem. $Q$ is the cost matrix that weights the current state variable and $R$ is the cost matrix that regulates the input $u$. The LQR provides a feedback controller represented by Koopman eigenfunction:

$$
u=-K_{\phi} \phi(y)
$$

which is linear in the eigenfunction, but generally nonlinear in original state $y$. We also consider the reference tracking, $u=-K_{\phi}\left(\phi\left(x-\phi_{\text {ref }}\right)\right)$, with a modified cost function. With this reference, we are allowed to control the Koopman eigenfunction into different levels. In our problem, the reference is determined through the zero velocity curve. Increasing the Jacobean integral corresponding to the system, we will have more total energy and thus satellite is allowed to fly to high potential energy space.

\section{Results and Discussion}

In this section, we are trying to solve a classical orbit transfer problem using the proposed Koopman reduced order control approach. To stimulate the practical application and to simplify the following description, we consider the earth to be the largest body in our restricted three body problem, the moon as the smaller 
planet, and our satellite as the third body. Using Equation (15), which describes a magnitude of thrust, we found a way to solve the minimal thrust orbit problem that allows satellite to transfer from the earth orbit to the moon orbit. In Figure 2 , red part is the original orbit that move around the moon and then transfer to the the orbit that move around the earth (blue line). This procedure requires that the total thrust force is minimal. The transfer procedure is illustrated in the green line. In Figure 3, it shows that from time 0 to 10 is the section when the orbital stays rotating around the earth (Blue dashed line in Figure 2), from time 10 to 15 is the section where the orbital changing into rotating around the moon (Green line in Figure 2), from time 15 to 30 is the section where the orbital rotate around the moon (Red dashed dot line in Figure 2).

As shown in Figure 3, between time 0 and 10, we kept the Jacobi integral low as a constant -3.19 to keep the orbit stay rotating around the earth. From time 10 to 15 , we increased the Jacobi integral to another constant -3.17 so that the orbital can switch to orbiting around the moon. At last, from time 15 to 30, we decreased the Jacobi back to the original constant -3.19 so that it will remain orbiting around the moon. Jacobi integral represents the total energy of the three body system; i.e., kinetic energy and potential energy. We control the orbit transfer through controlling the Jacobi integral base on the zero velocity curves (Figure 1), as zero velocity curve represents the region that satellite can fly. These two constants -3.19 and -3.17 corresponds to the zero velocity curve that pass through Lagrangian point $L_{1}$ and $L_{2}$, respectively (with a minus sign difference).

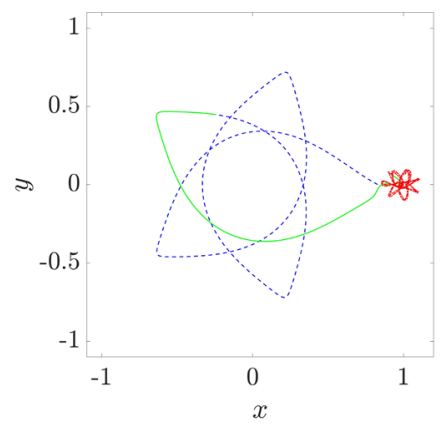

Figure 2. Orbital curve of the third body (satellite). Blue dashed line: from time 0 to 10 when the satellite stays rotating around the earth. Green line: from time 10 to 20 when the orbital transfers into the orbit rotating around the moon. Red dashed dot line: from time 15 to 30 when the orbital stays rotating around the moon.

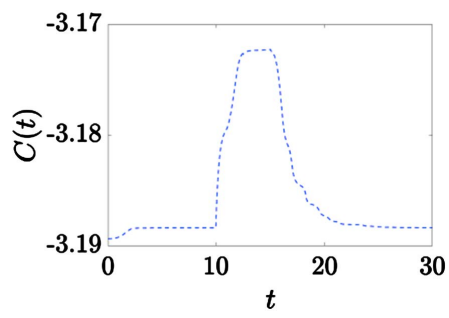

Figure 3. Time-history of Jacobean Integral when transferring from the earth to moon. 
Figure 4 and Figure 5 shows us the time history of feedback gain for control in $x$ and $y$ direction, respectively. Although the controller we design is linear for Jacobi Integral (16), but the controller is nonlinear with respective to original state variable; i.e., velocity and displacement in $x$ and $y$ directions. The advantage of Koopman controller is that we are allowed to combine Koopman eigenfunction and traditional linear controller to design a nonlinear controller easily. Using Jacobi Integral (total energy) as the Koopman eigenfunction, it provides us a way to control the orbit and perform the orbit transfer easily.

Furthermore, we modify the mass ratio $\mu$ in circle restricted three body problem to consider the sun-earth-moon system, assuming that the force of the moon and other planets are negligible. In that the orbit moving around the earth is extremely small compared to the earth-sun distance, we also show results that zoom in to the region around the earth (Figure 7). In Figure 6 and Figure 7, red part is the original orbit that moves around the moon and then transfer to the orbit that moves around the earth (blue line). This procedure requires that the total thrust force is minimal. The transfer procedure is illustrated in the green line. In Figure 8, it shows that from time 0 to 10 is the section where the orbital stays rotating around the earth (Blue dashed line in Figure 6 and Figure 7), from time 10 to 15 is the section where the orbital changing into rotating around the moon (Green line in Figure 6 and Figure 7), from time 15 to 100 is the section where the orbital rotate around the moon (Red dashed dot line in Figure 6 and Figure 7).

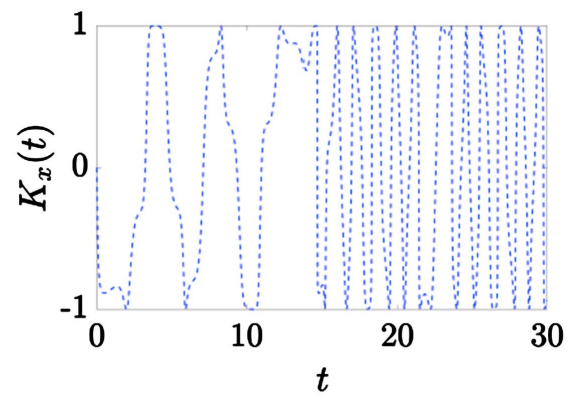

Figure 4. Time history of feedback gain for control in $x$ direction when transferring from the earth to moon.

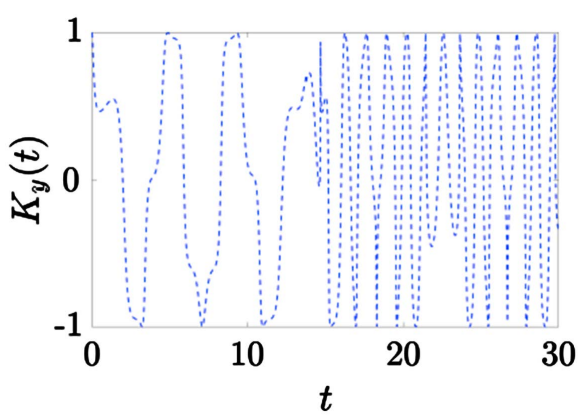

Figure 5. Time history of feedback gain for control in $y$ direction when transferring from the earth to moon. 


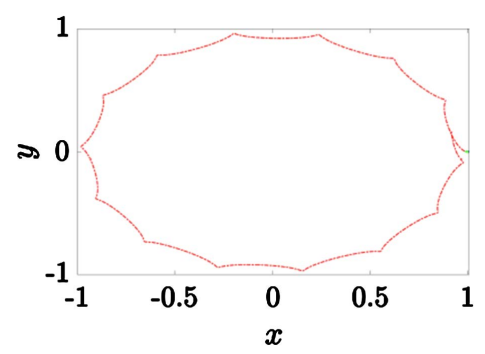

Figure 6. Orbital curve of the satellite. Blue dashed line: from time 0 to 10 when the satellite stays rotating around the earth. Green line: from time 10 to 15 when the orbital transfers into the orbit rotating around the sun. Red dashed dot line: from time 15 to 100 when the orbital stays rotating around the sun.

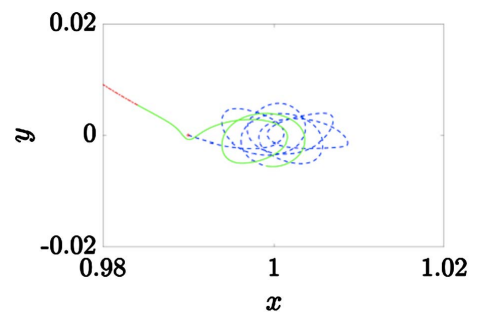

Figure 7. Orbital curve of the satellite, zoomed in around the area around the earth. Blue dashed line: from time 0 to 10 when the satellite stays rotating around the earth. Green line: from time 10 to 15 when the orbital transfers into the orbit rotating around the sun. Red dashed dot line: from time 15 to 100 when the orbital stays rotating around the sun.

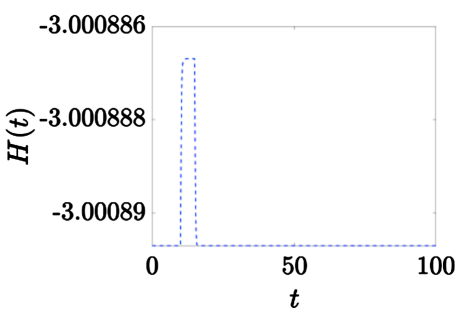

Figure 8. Time-history of Jacobean Integral when transferring from the earth to sun.

As shown in Figure 8, between time 0 and 10, we kept the Jacobi integral low as a constant -3.0008906 to keep the orbit stay rotating around the earth. From time 10 to 15 , we increased the Jacobi integral to another constant -3.000886 so that the orbital can switch to orbiting around the moon. At last, from time 15 to 100 , we decreased the Jacobi back to the original constant -3.0008906 so that it will remain orbiting around the moon. Jacobi Integral represents the total energy of the three body system; i.e., kinetic energy and potential energy. We control the orbit transfer through controlling the Jacobi integral base on the zero velocity curves (Figure 1), as zero velocity curve represents the region that satellite can fly. These two constants -3.0008906 and -3.0008866 corresponds to the zero velocity curve that pass through Lagrange point $L_{1}$ and $L_{2}$, respectively (with a minus sign difference).

Figure 9 and Figure 10 show us the time history of feedback gain for control in $x$ and $y$ direction while moving towards the sun, respectively. Although the 


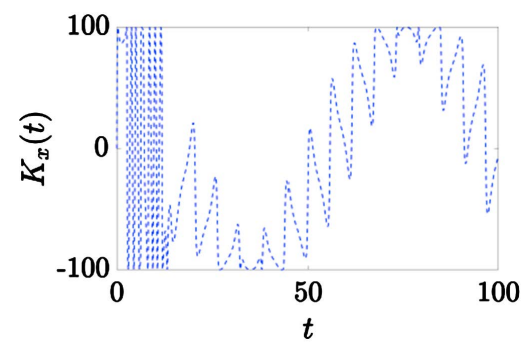

Figure 9. Time history of feedback gain for control in $x$ direction when transferring from the earth to sun.

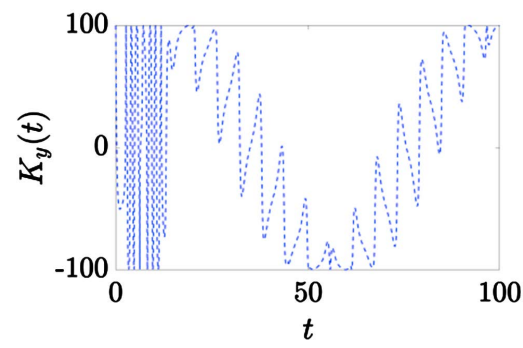

Figure 10. Time history of feedback gain for control in $y$ direction when transferring from the earth to sun.

controller we design is linear for Jacobi Integral (16), but the controller is nonlinear with respective to original state variable; i.e., velocity and displacement in $X$ and $y$ directions.

\section{Conclusion}

In this paper, we use a Circle Restricted Three-Body Problem (CRTBP) to simulate the system among the earth, moon and satellite and the system among the sun, earth and satellite. Then we reformulate this problem with controller into the description using Koopman eigenfunction. Although original dynamical system is nonlinear, the Koopman eigenfunction behaves linearly. Choosing Jacobi integral as the Koopman eigenfunction and using the zero velocity curve as the reference for control, we are allowed to combine traditional Linear Quadratic Regulator (LQR) controller to design a nonlinear controller. At last, we design the low thrust orbit transfer strategy for the satellite flying from the earth to the moon or from the earth to the sun.

\section{Conflicts of Interest}

The author declares no conflicts of interest regarding the publication of this paper.

\section{References}

[1] Bertsekas, D.P. (1999) Nonlinear Programming. Athena Scientific Belmont.

[2] Brunton, S.L., Brunton, B.W., Proctor, J.L. and Kutz, J.N. (2016) Koopman Invariant Subspaces and Finite Linear Representations of Nonlinear Dynamical Systems for Control. PLoS ONE, 11, e0150171.

https://doi.org/10.1371/journal.pone.0150171 
[3] Brunton, S.L, Proctor, J.L. and Kutz, J.N. (2016) Discovering Governing Equations from Data by Sparse Identification of Nonlinear Dynamical Systems. Proceedings of the National Academy of Sciences of USA, 113, 3932-3937. https://doi.org/10.1073/pnas.1517384113

[4] Fahroo, F. and Ross, I.M. (2002) Direct Trajectory Optimization by a Chebyshev Pseudospectral Method. Journal of Guidance, Control, and Dynamics, 25, 160-166. https://doi.org/10.2514/2.4862

[5] Herman, A.L. and Conway, B.A. (1996) Direct Optimization Using Collocation Based on High-Order Gausslobatto Quadrature Rules. Journal of Guidance, Control, and Dynamics, 19, 592-599. https://doi.org/10.2514/3.21662

[6] Kaiser, E., Kutz, J.N. and Brunton, S.L. (2017) Data-Driven Discovery of Koopman Eigenfunctions for Control. arXiv preprint arXiv:1707.01146

[7] Koopman, B.O. (1931) Hamiltonian Systems and Transformation in Hilbert Space. Proceedings of the National Academy of Sciences of USA, 17, 315-318. https://doi.org/10.1073/pnas.17.5.315

[8] Topputo, F. and Zhang, C. (2014) Survey of Direct Transcription for Low-Thrust Space Trajectory Optimization with Applications. Abstract and Applied Analysis, 2014, Article ID 851720. 